\title{
BMJ Open Qualitative exploration of the benefits of group-based memory rehabilitation for people with neurological disabilities: implications for rehabilitation delivery and evaluation
}

\author{
Niki Chouliara, ${ }^{1}$ Nadina B Lincoln ${ }^{2}$
}

To cite: Chouliara N, Lincoln NB. Qualitative exploration of the benefits of group-based memory rehabilitation for people with neurological disabilities: implications for rehabilitation delivery and evaluation. BMJ Open 2016;6:e011225. doi:10.1136/bmjopen-2016011225

- Prepublication history for this paper is available online. To view these files please visit the journal online (http://dx.doi.org/10.1136/ bmjopen-2016-011225).

Received 22 January 2016 Revised 3 May 2016 Accepted 11 July 2016

CrossMark

${ }^{1}$ NIHR Collaboration of Leadership in Applied Health Research and Care, Institute of Mental Health, University of Nottingham, Nottingham, UK

2Department of Rehabilitation and Ageing, School of Medicine, University of Nottingham, Queen's Medical Centre, Nottigham, UK

Correspondence to Dr Niki Chouliara;

niki.chouliara@nottingham.ac.uk

\section{ABSTRACT}

Objective: To identify patient-perceived benefits of memory rehabilitation and draw transferrable lessons for the delivery and evaluation of similar interventions for people with neurological disabilities.

Methods: A qualitative study was conducted as part of a pragmatic randomised controlled trial comparing 2 memory rehabilitation approaches with a self-help control group. Postintervention interviews were conducted with 20 participants with a diagnosis of traumatic brain injury, multiple sclerosis or stroke. Data were analysed using a qualitative content analysis approach.

Results: Participants receiving memory rehabilitation reported that the sessions responded to previously unmet needs for information on brain injury and memory function and developed their insight along with a sense of self-efficacy and control over the management of their memory problems. Although they did not experience major improvements in their memory function per se, they reported that rehabilitation gave them the skills to effectively cope with the residual deficits. Respondents in the control groups did not report similar benefits. The opportunities for interaction offered by the group setting were greatly valued by all respondents. Mixed aetiology groups were received positively; however, marked differences in cognitive performance were frustrating for some participants.

Conclusions: The study highlighted important patient-perceived outcomes that should be considered by researchers and rehabilitation professionals when evaluating the effects of memory rehabilitation. The use of domain-specific outcome measures which reflect these areas is recommended. Qualitative changes in the use of memory aids may be achieved which cannot be captured by frequency indices alone. The benefits of the group-based rehabilitation approach were stressed by participants, suggesting that a combination of group and individual sessions might be a good practice.

Trial registration number: ISRCTN92582254; Results.

\section{Strengths and limitations of this study}

- The study offers an in-depth exploration of patient-perceived outcomes of memory rehabilitation for people with neurological disabilities, and uncovers benefits in domains unaccounted for by previous research.

- The study design enabled the identification of effects specific to the memory rehabilitation interventions as compared with the self-help control programme.

- The findings have implications for delivering and evaluating memory rehabilitation interventions, and relevant recommendations to clinicians and researchers are provided.

- The transferability of the findings should be examined in relation to the specific aims and goals of future rehabilitation programmes. It is suggested that our recommendations should be relevant to rehabilitation interventions that adopt an integrative approach targeting not only memory difficulties but also their effects on patient awareness, mood and coping style.

\section{INTRODUCTION}

Memory rehabilitation is a promising approach to the management of memory problems in people with acquired brain injury (BI). The aims of cognitive rehabilitation can be understood under the framework introduced by the $\mathrm{WHO}^{1}$ which classifies the effects of BI into 'impairment' (eg, memory function), 'activity limitations' (eg, memory failures) and 'participation' (eg, social, professional life). It is important that all these levels of description be considered when developing and evaluating rehabilitation interventions. Memory rehabilitation efforts, particularly in the postacute stage, are mainly focused on developing patients' ability to cope with or compensate for residual 
memory deficits, as well as promoting participation rather than restoring memory impairment per se. ${ }^{23}$

Research to date has produced mixed findings on the effectiveness of memory rehabilitation interventions, which engenders questions about the way outcome is assessed. $^{4}{ }_{5}$ A number of factors make the evaluation and interpretation of rehabilitation outcomes a challenging task. Rehabilitation involves complex multicomponent interventions which often interact with a range of personal and contextual factors to affect outcome. ${ }^{6}$ Changes in thought and behavioural patterns are required, which may result in individual differences regarding when and how people experience the effects of rehabilitation. ${ }^{7}$

The quality and appropriateness of the measures employed has been highlighted as one of the main determinants of the outcome of rehabilitation. ${ }^{8}{ }^{9}$ The majority of studies have evaluated the success of memory rehabilitation by means of formal psychometric tests (eg, Wechsler memory scales). These provide robust information on the nature and severity of impairment but not on the way it affects patients' daily lives or the use of compensatory mechanisms. ${ }^{6}$ In clinical practice, it is suggested that standardised measures should be complemented by behavioural assessments, including self-report measures (ie, questionnaires, checklists and diaries), observations and interviews. ${ }^{3}$ These allow rehabilitation professionals to obtain information on relevant functional outcomes as well as patients' perceptions of their problems.

A wide range of self-report questionnaires has been used in research studies to evaluate the subjective experience of living with memory problems. Some of the most commonly used questionnaires in research studies ${ }^{10-12}$ have been developed for healthy older adults and emphasise issues which may not represent rehabilitation priorities. Their length, complexity of wording and response format may further undermine the ability of cognitively impaired individuals to accurately report on their memory failures. Furthermore, the content of some questionnaire items may limit their responsiveness and sensitivity to subtle improvements following memory rehabilitation. $^{13}$

Qualitative interviews offer an advantage over questionnaires as they allow patients to describe their experience in their own words and at their own pace. They, therefore, present an opportunity for researchers to re-examine their own assumptions on the effects of an intervention and identify subtle but potentially important improvements which might be missed when relying exclusively on quantification. ${ }^{14}{ }^{15}$ The UK Medical Research Council framework on complex interventions recommends the use of qualitative evaluations alongside randomised controls trials (RCTs) in order to explore the value of the intervention to service users, facilitate the interpretation of the results, and obtain a better understanding of how the intervention worked or why it did not work and how it could be improved. ${ }^{8}{ }^{16}$ This information will be crucial in developing strategies to promote the implementation of the intervention in clinical practice and ensure it meets patients' needs. ${ }^{17}$

The use of postintervention interviews is becoming increasingly popular; however, few studies in memory rehabilitation have sought qualitative feedback from their participants. Where participants' accounts were obtained, important gains were noted, with rehabilitation reportedly promoting self-awareness, expanding their knowledge on memory functioning and improving the use of compensatory strategies. ${ }^{18-20}$ Interestingly, these improvements were not captured by the quantitative measures used in the studies. One explanation could be that the interventions were ineffective and participants' views reflected inaccurate self-appraisals or attempts to please the researchers. Another possibility, suggested by the authors of these studies, was that participants did experience some meaningful improvements which were not detected by the quantitative outcome measures. Owing to methodological limitations in obtaining and presenting patients' feedback, the above studies do not provide sufficient information to substantiate this hypothesis and draw recommendations on how to improve outcome evaluation in future memory rehabilitation studies. Another problem in interpreting the findings was the lack of control groups which did not permit differentiating the effects of the interventions from other possible influences.

There is a lack of rigorous qualitative work which will derive rich descriptions of patients' experiences and allow for topics important to them to emerge unprompted. The present study examined the postintervention interviews of patients with neurological disabilities participating in the main phase of a memory rehabilitation RCT. $^{21}$ The trial compared the effectiveness of two types of memory rehabilitation programmes, focusing on either 'compensation' or 'restitution' orientated strategies, with a self-help control programme on memory functioning, ${ }^{11}{ }^{22}$ use of memory aids, ${ }^{23}$ $\operatorname{mood}^{24} 25$ and independence in activities of daily living. ${ }^{26}$ We examined the interviews of participants in the two intervention programmes in relation to those in the self-help programme in order to: (1) explore their views on the programme they attended and identify perceived benefits following memory rehabilitation, and (2) examine whether participants' accounts allow drawing transferrable lessons for the delivery and evaluation of future memory rehabilitation programmes.

\section{METHODOLOGY}

\section{Study design and participants}

This is a qualitative interview study conducted within the main phase of the ReMIND trial (Rehabilitation of Memory in Neurological Disabilities). ${ }^{21}$ The philosophical underpinnings of the broader study were grounded in pragmatism, which provided a framework for the integration of qualitative and quantitative findings. Within 
this research paradigm, methodological tools can be combined flexibly in order to best answer the research questions and draw findings transferrable in clinical practice. $^{27} 28$

Participants with memory problems following traumatic BI (TBI), stroke and multiple sclerosis (MS) were randomly allocated (in blocks of four) to one of three programmes: a compensation-based memory rehabilitation programme, a restitution-based programme or a self-help control programme. The use of a mixed aetiology sample was preferred in order to reflect the reality of clinical services where the provision of memory rehabilitation is not done on the basis of the diagnosis. Information about the study was sent to potential referrers working in hospitals and/or rehabilitation centres, general practice services, and stroke, MS and TBI services. Only community-based patients were considered for recruitment. The programmes were delivered on a group basis, although participants received an introductory one-to-one session. Individual sessions were also offered during the course to those participants who missed a group meeting. The groups were conducted at the University of Nottingham and Derby Royal Hospital and were delivered by either one of two research assistants trained by the clinical psychologist leading the study. The three programmes corresponded to integral components of memory rehabilitation interventions that are usually offered in clinical practice. The content of the progammes, documented in a training manual for replicability purposes, was informed from the literature, clinicians' recommendations and pre-existing workbooks. ${ }^{29}$ A summary description of the trial design is given in box 1 .

Participants in the interview study attended the programmes run between May 2007 and July 2008. Twenty-four people were randomised to two compensation, two restitution and two self-help groups with four people allocated in each group. Two participants dropped out of the first restitution group, after attending two and three sessions, respectively. One of them had a relapse of MS and the other one decided not to continue coming to the groups. Interviews were conducted 7 months after randomisation (October 2007 to February 2009) at the University of Nottingham and Derby Royal Hospital.

\section{Interviews}

One-to-one semistructured interviews were conducted, to allow participants to express their views unaffected by the presence of other group members. It was considered that the flexibility and non-directive style of the semistructured interview process promoted conversational communication and provided a context where recall could be facilitated. ${ }^{30}$ The schedule was kept purposely broad to elicit unprompted answers. The questions were not fixed but a list of topics was developed to serve as a guide. These topics were informed by the therapeutic goals of holistic rehabilitation programmes ${ }^{2}$ as well as
Box 1 Summary description of the randomised controlled trial

Overall design: single (patient) blind randomised controlled trial. Random allocation: random allocation in cohorts of four to compensation, restitution or self-help groups.

Groups

Location: University of Nottingham/Derby City Hospital.

Format: multiweek, group-based rehabilitation programmes.

Number of sessions: 1 introductory individual session and 10 group sessions (1 session per week).

Length of each session: $1 \frac{1}{2}$ hours with a $10-15$ min break.

Number of participants in each group: four.

Target population:

Diagnosis: people with brain injury due to traumatic brain injury, multiple sclerosis and stroke.

Age: 18 years of age and older.

Level of memory impairment: overall profile score of $<3$ on the Rivermead Behavioural Memory Test-Extended version or $<25$ th centile on the Doors and People test.

1. Focus of restitution programme: training in use of internal memory aids, attention training techniques, repeated practice and extended rehearsal.

2. Focus of compensation programme: training in use of external memory aids and ways of adapting to memory problems.

3. Goals of self-help control programme: emotion-focused programme, memory training not offered, encourage participants to discuss emotional issues related to impairments, practice relaxation exercises (eg, Jacobson's Progressive Muscular Relaxation).

Outcome evaluation:

(a) Quantitative measures:

- Memory functioning: Everyday Memory Questionnaire, Rivermead Behavioural Memory Test-Extended Version;

- Use of memory aids: Internal and External Memory Aids Questionnaires;

- Mood: General Health Questionnaire-12, Wimbledon Self Report Scale;

- Independence in activities of daily living: Nottingham Extended Activities of Daily Living Scale.

(b) Postintervention qualitative interviews with participants.

Follow-up assessment points: 5 and 7 months after randomisation.

Feedback interviews: 7 months after randomisation.

the preliminary findings of the pilot phase of the ReMIND study and they included: patients' experience of memory failures in everyday life prior and following participation in the study, use of memory strategies, effects of the intervention on their personal, professional and social life, perceived changes in cognitive function, coping style and mood, thoughts on the quality and delivery format of the sessions, and suggestions for improvements. An opening question asked participants to indicate the most beneficial aspects of the programme they attended. Open-ended and follow-up questions were employed in order to obtain breadth and depth in the responses. New topics introduced by respondents were also discussed as they arose. One of 
the two interviewers (NBC) attended some of the group sessions as an observer. To address any possible bias related to participants' prior knowledge of the interviewer, half of the interviews were conducted by a researcher who was not involved with any other aspects of the trial. Guidelines were developed for both researchers to follow in order to promote consistency in interviewing. Prior to the interviews, it was explained to participants that their feedback, either positive or negative, could help researchers improve the rehabilitation programme. Information on their treatment allocation was given to participants at the end of the interviews. Interviews lasted between $40 \mathrm{~min}$ and 1 hour, and they were audio recorded, transcribed verbatim and anonymised.

\section{Analysis}

Data were analysed following a qualitative content analysis procedure ${ }^{31}$ to examine the latent content of the narratives and provide an indication of the prevalence of the identified categories. The coding scheme was developed inductively and was semantically as near as possible to the narratives in order to gain direct information from participants without imposing any preconceived categories. ${ }^{32}$ A constant comparison process was applied, continuously comparing the views of respondents within the same programme and across the three programmes to identify commonalities and exceptions, derive data-driven codes and group them into core categories sharing a similar meaning. This process was considered the most appropriate to make the differences between the three programmes apparent and highlight topics specific to the two intervention programmes. Codes were assigned neutral labels that described directly the content of the text excerpt. In some cases, theoretical concepts were also employed in order to synthesise and label the main categories. For example, the term 'control beliefs' was used to group patients' perceptions of self-efficacy and control in relation to the management of their memory problems. ${ }^{33}$

Data were analysed by the first author. To enhance the credibility of the coding scheme, an independent researcher, not involved with any other aspects of the study, reviewed a random sample of $50 \%$ of the interviews. Using a coding manual with definitions of the identified categories, the researcher assessed whether the codes accurately captured respondents' meanings without excluding important information. ${ }^{34}$ Data were organised electronically using a qualitative data analysis software package (QSRNVivo V.8, QSR International) The demographic characteristics of the sample were evaluated using the SPSS statistical package V.19.0.

\section{RESULTS}

Interviews were conducted with 20 participants of the 24 who were allocated to groups. Of the 22 participants who had completed the programme, 2 participants could not be reached and 1 participant could not continue participation due to poor health (MS relapse). One of the two participants who had dropped off the restitution programme agreed to be interviewed. The demographic and psychometric characteristics of interviewees are shown in table 1.

Across all the three groups, participants had an average overall profile of 1 on the Rivermead Behavioural Memory Test-Extended Version, signifying poor memory. ${ }^{22}$ Based on their scores on the Sheffield Screening Test for Acquired Language Disorders, ${ }^{36}$ they did not experience receptive or expressive language problems. Kruskal-Wallis analyses of variance showed no significant differences between the three programmes on age, time since injury/diagnosis and psychometric characteristics. It was not possible to examine differences on gender and diagnosis as more than $25 \%$ of cells had an expected frequency of less than five cases.

\section{Identified categories}

The second coder confirmed that the identified categorical scheme was valid across the entire data set and accurately described participants' narratives. Participants' unprompted responses to the opening question indicated the most beneficial aspects of the memory rehabilitation programmes to be perceived improvements in: (1) memory knowledge, (2) use of memory aids, and (3) self-efficacy towards the management of memory problems. For the self-help group, the most helpful components were: (1) practising self management strategies, and (2) developing a more proactive stance and becoming more organised in their efforts to cope with neurological disabilities. The most frequently cited benefit common across the three programmes was the experience of being in a group of people with similar problems. Responses to the opening question were also found to represent repeated patterns running through patients' narratives and forming the core categories. Individual cases that contradicted the themes or conveyed an interesting idea were also incorporated in the report to reflect the range of different views in the data set. The interviews of participants in the self-help programmes were analysed to allow the identification of benefits unique to memory rehabilitation. However, in order to provide rich descriptions of patients' experiences of memory rehabilitation, the results presented here focus on the responses of those in the two intervention programmes. To highlight the differences between the three programmes, the number of participants who reported benefits in relation to each category is also shown in table 2.

\section{Memory knowledge and self-awareness}

This category refers to respondents' understanding of how memory works ${ }^{37}$ and represented one of the most beneficial aspects of memory rehabilitation according to participants in the two intervention programmes. 
Table 1 Demographic and psychometric characteristics of participants

\begin{tabular}{|c|c|c|c|c|}
\hline & \multicolumn{4}{|l|}{ Group } \\
\hline & Compensation $(\mathrm{n}=7)$ & Restitution $(n=6)$ & Self-help $(n=7)$ & p Value* \\
\hline \multicolumn{5}{|l|}{ Gender } \\
\hline \multicolumn{5}{|l|}{ Female } \\
\hline $\mathrm{n}$ & 4 & 3 & 2 & - \\
\hline Per cent & 57.1 & 50.0 & 28.6 & \\
\hline \multicolumn{5}{|l|}{ Male } \\
\hline $\mathrm{n}$ & 3 & 3 & 5 & \\
\hline Per cent & 42.9 & 50.0 & 71.4 & \\
\hline \multicolumn{5}{|l|}{ Diagnosis } \\
\hline \multicolumn{5}{|l|}{ TBI } \\
\hline $\mathrm{n}$ & 4 & - & 1 & - \\
\hline Per cent & 57.1 & - & 14.3 & \\
\hline \multicolumn{5}{|l|}{ MS } \\
\hline $\mathrm{n}$ & 3 & 5 & 2 & \\
\hline Per cent & 42.9 & 83.3 & 28.6 & \\
\hline \multicolumn{5}{|l|}{ Stroke } \\
\hline $\mathrm{n}$ & - & 1 & 4 & \\
\hline Per cent & - & 16.7 & 57.1 & \\
\hline \multicolumn{5}{|l|}{ Age } \\
\hline Median & 43.0 & 52.0 & 58.0 & 0.13 \\
\hline $\mathrm{IQR}^{*}$ & $35.0-55.0$ & $37.0-54.25$ & $41.0-65.0$ & \\
\hline \multicolumn{5}{|c|}{ Time since injury/diagnosis (number of months) } \\
\hline Median & 73.0 & 96.0 & 75.5 & 0.20 \\
\hline IQR & $20.3-120.0$ & $37.7-150.0$ & $37.30-110.0$ & \\
\hline \multicolumn{5}{|c|}{ NART estimated IQ score } \\
\hline Median & 107.4 & 102.0 & 110.0 & 0.28 \\
\hline IQR & $98.3-115.3$ & $96.7-113.5$ & $100.0-122.0$ & \\
\hline \multicolumn{5}{|c|}{ RBMT-E overall profile score } \\
\hline Median & 1.0 & 1.0 & 1.0 & 0.48 \\
\hline IQR & $1.0-2.0$ & $0-1.0$ & $0.25-1.75$ & \\
\hline \multicolumn{5}{|c|}{ SSTALD total score } \\
\hline Median & 20.0 & 19.0 & 19.0 & 0.51 \\
\hline IQR & 19.0-20.0 & 17.0-20.0 & 19.0-20.0 & \\
\hline \multicolumn{5}{|c|}{ GHQ-30 total score } \\
\hline Median & 36.0 & 20.0 & 35.0 & 0.12 \\
\hline IQR & $22.0-56.0$ & $10.0-30.5$ & $20.5-39.0$ & \\
\hline
\end{tabular}

It was the first time I got scientific explanations of what I' $\mathrm{m}$ going through so I thought it was quite fascinating actually. (Male, compensation, TBI)

This aspect of the intervention reportedly addressed an unmet need for information on the cognitive aftermath of BI. With the focus of their care being on physical impairments, participants felt that the cognitive consequences of BI were often being overlooked by healthcare professionals. One woman with MS described how her memory symptoms had been attributed to mood disturbances and 'mental health difficulties'.

It is difficult and painful to hear myself described in these terms by those who are paid to support me. (Female, compensation, MS)
Respondents viewed the provision of information and feedback on their performance, along with their interaction with other group members, as mechanisms triggering a process of self-reflection and enabling a more realistic appraisal of the nature and severity of their memory problems. At the same time, the groups reportedly functioned as a supportive context, normalising patients' experience and promoting acceptance.

Coming to the groups made me more aware of the level of my memory whereas I used to think I have a good memory. (Male, compensation, MS)

I used to think they are quite bad you know...because I didn't talk to anyone but now I don't feel I' $m$ that bad as I thought I was. (Female, restitution, TBI) 
Table 2 Summary of identified categories representing patient-perceived benefits

\begin{tabular}{|c|c|c|c|c|c|c|c|c|}
\hline \multirow[b]{2}{*}{ Categories } & \multicolumn{2}{|c|}{$\begin{array}{l}\text { Compensation } \\
(n=7)\end{array}$} & \multicolumn{2}{|c|}{$\begin{array}{l}\text { Restitution } \\
(n=6)\end{array}$} & \multicolumn{2}{|c|}{ Self-help $(n=7)$} & \multicolumn{2}{|c|}{ Total $(\mathrm{N}=20)$} \\
\hline & $\bar{n}$ & Per cent & $\bar{n}$ & Per cent & $\bar{n}$ & Per cent & $\overline{\mathbf{n}}$ & Per cent \\
\hline Memory knowledge & 7 & 100 & 5 & 83.3 & 1 & 14.3 & 13 & 65 \\
\hline Self-awareness & 5 & 71 & 4 & 67 & 0 & 0 & 9 & 45 \\
\hline Severity of MP & 5 & 71 & 4 & & & & 5 & 25 \\
\hline Cognitive strengths and weaknesses & 3 & 43 & 3 & 50 & & & 6 & 30 \\
\hline Anticipatory awareness & 4 & 57 & 2 & 33 & & & 6 & 30 \\
\hline Memory aids & 6 & 86 & 5 & 83 & 0 & 0 & 11 & 55 \\
\hline Learning new strategies & 4 & 57 & 4 & 67 & & & 8 & 40 \\
\hline Use strategies effectively & 6 & 86 & 5 & 83 & & & 11 & 55 \\
\hline Cognitive function & 3 & 43 & 4 & 67 & 1 & 14.3 & 8 & 40 \\
\hline Control beliefs & 6 & 86 & 4 & 67 & 0 & 0 & 10 & 50 \\
\hline Memory self-efficacy & 6 & 86 & 4 & 67 & 0 & 0 & 10 & 50 \\
\hline Feeling in control & 5 & 71 & 3 & 50 & 2 & 9 & 10 & 55 \\
\hline $\begin{array}{l}\text { Being proactive towards } \\
\text { management of problems }\end{array}$ & 5 & 71 & 4 & 67 & 3 & 43 & 12 & 60 \\
\hline Emotional adjustment & 4 & 57 & 3 & 50 & 6 & 86 & 13 & 65 \\
\hline Stress related to MP & 4 & 57 & 3 & 50 & 2 & 29 & 9 & 45 \\
\hline General mood status & 1 & 14 & 1 & 17 & 6 & 86 & 8 & 40 \\
\hline Disclosure & 3 & 43 & 2 & 33 & 2 & 29 & 7 & 35 \\
\hline Benefits of the group setting & 7 & 100 & 6 & 100 & 6 & 86 & 19 & 95 \\
\hline
\end{tabular}

Some respondents noted that rehabilitation changed their perception of memory as a unitary function and guided them in distinguishing between different memory processes. The idea that memory impairment is not necessarily generalised but can be limited to specific components was a powerful message, which shifted the focus of attention from participants' difficulties to their strengths.

It made me look at which one you were doing better than other and which one you were doing badly and you need to try to improve on. (Male, compensation, TBI)

Anticipatory awareness, or the ability to recognise in advance the circumstances within which memory failures are likely to occur, ${ }^{38}$ was another area in which participants in the intervention programmes reported improvements. They described the sessions as an opportunity to examine different real-life scenarios that challenged their memory and explore whether they could be anticipated and managed.

It was very useful identifying the bits I've struggled with because then it made me more aware if I' $\mathrm{m}$ catching that type of scenario in real life then I needed to make more effort. (Female, compensation, TBI)

Not everyone felt emotionally prepared to acknowledge the severity of their memory difficulties. One participant described how she was reminded of her physical and cognitive losses at a time when she wanted to move on in her life.
I didn't keep reading the information. I think in a way I put the accident behind me so I don't want to keep thinking. (Female, compensation, TBI)

In his narrative, one participant who had dropped off the restitution programme reported that understanding the severity of his difficulties was a distressing process in which he was reluctant to engage. He was worried that what he could discover about the level of his cognitive abilities would heighten his anxiety about the future. He added that he would have, instead, preferred to receive some reassurance on the progression of his condition which was, however, out of the scope of the programmes.

See, I do worry where my memory is going to end up. While I am well, I am holding on to not knowing...the day I know I get worried. I don't want to realise that. The groups were all about programming your memory. You don't know what you are going to forget. I'd like to know if that will carry on going. (Male, restitution, MS)

On the contrary, some other participants reported that increasing their understanding of memory deficits was an important step towards their successful management and, therefore, they would have valued more information.

Other members of the group might have needed not quite the detail of that, while myself, I wanted a bit more information. (Male, compensation, MS) 


\section{Memory aids}

The use of memory aids was reported as a key area of improvement in the intervention programmes, with participants feeling more effective in their efforts to compensate for their memory problems. Most respondents were new to the idea of internal memory aids (eg, visual imagery, etc) and errorless learning but were already familiar with external memory aids (eg, diaries, alarms, etc). Apart from expanding their repertory of memory aids, participants re-evaluated the way they used the ones they had in place.

It has given me tools to use which I haven't thought of or if I have been doing I hadn't really realised I was doing it until I actually focused on what I'm doing, like "chunking”. Because I'm more conscious that I can do it, I actually use that a lot more now than I did before. (Female, compensation, TBI)

Not all strategies worked for everyone and some respondents stressed the importance of acknowledging this within the groups. Rehabilitation offered them a context where they could explore a range of different options and identify the most suitable strategies for their individual needs or learn to adapt them accordingly.

Some of the techniques helped, some didn't and it was nice to know that the ones that didn't work for me didn't necessarily work for others. (Female, restitution, TBI)

\section{Cognitive function}

Overall, there was little evidence in the interviews to suggest that participants experienced improvements in their memory function following rehabilitation. The exception was two participants who felt their ability to remember information had improved as a result of the sessions. Their confidence was grounded on the systematic use of internal memory aids which allowed them to capitalise on their cognitive strengths.

I used to carry a little Dictaphone, a little tape recorder and I used it quite a lot...I use it less and less these days. If I have to remember something then I commit it to my verbal memory, which before I couldn't. Instead of using the Dictaphone I would just say it out loud so I can hear it, so I relocate the information. (Male, compesation, MS)

A degree of uncertainty about the effect of rehabilitation on their memory function was evident in the interviews of some participants with TBI. They explained that, being still at an early stage postinjury, they either had not yet returned to their old routines or they found it difficult to isolate the contribution of rehabilitation from other factors, such as spontaneous recovery. Participants were also asked whether they had noticed any changes in relation to the memory failures they had reported at the introductory session. Where improvements were mentioned, they were mainly in relation to prospective memory tasks.
Appointments have improved because I use the alarm on my phone, and I try and write more things in my diary than I ever did before. (Female, compensation, MS)

As part of their training participants received information on the role that other functions, such as attention, have in supporting memory performance. As a result, they reported making a more conscious effort to reduce distractions and actively pay attention to the information they wanted to memorise.

I used to think that memory is just remembering something; it's not actually. I found that one of the problems for me was that I wasn't making that effort to concentrate. (Female, compensation, TBI)

\section{Control beliefs}

Participants drew a distinction between actual memory ability, where no major benefits were noted, and the belief that there are ways to prevent forgetting using the strategies they were taught. In contrast to participants in the self-help programme, the majority of participants in the two intervention programmes felt more confident in their ability to effectively cope with memory difficulties.

I feel more confident that I will manage, cause if I've got a problem I know I've got ways to deal with it. I can look back at what we did in the group sessions and try different memory aids. (Male, compensation, TBI)

Setting realistic goals in line with their abilities, and becoming increasingly skilful in the use of memory strategies were two factors identified by participants as important in enhancing their memory self-efficacy.

I' $m$ no different. I remember things better because I do the exercises and I check things and I make sure I take a note of this and I got an idea what to do to help myself. (Female, restitution, MS)

In their narratives, memory rehabilitation was described as an empowering experience which contributed to them becoming more independent in performing memory tasks and regaining a sense of control in their lives.

I think I try and do it myself rather than straight away shout to my husband to look. If my husband is driving I used to just totally rely on him, where he parked and just follow him. But now I just make a point of knowing where the car is parked...I found that I'm more independent. (Female, compensation, TBI)

Participants from all the three programmes appeared motivated to take a more proactive approach to the management of their problems. Within the intervention programmes, being introduced to the concept of neuroplasticity was identified by respondents as a key driver to action and engagement in activities beneficial to their cognitive functioning. 
I' $\mathrm{m}$ aware now that exercising the brain is good. I just look for challenging situations. I started doing one of these brain training games. I got a memory one, and I got a vocabulary one... and I think I am improving with practice. (Female, compensation, TBI)

\section{Emotional adjustment}

Perceived benefits in participants' ability to effectively manage stress formed a dominant theme, common across the three programmes. Respondents in the selfhelp programme attributed this effect to the practice of relaxation techniques, whereas participants receiving rehabilitation identified the effective use of memory strategies as the main contributing factor. Another helpful element was the recognition of the reciprocal relationship between memory function and anxiety with participants reportedly making a conscious effort to reduce their stress levels.

If I forget I just take my time and eventually it comes back to me. I know it still lets me down, but I'm now more able to accept that if I stop panicking words will come back to me, names will come back to me, events will come back to me. (Female, compensation, TBI)

Their efforts to control stress levels were also supported by improvements in planning and goal setting. As a result, participants attempted to strike a balance between their abilities and environmental demands by setting small and achievable goals, as well as negotiating other peoples' expectations on them.

\section{I always used to say yes to things and since coming to the sessions it has made me look at things slightly differ- ently...the sessions did actually teach me to say stop, step back a little bit and don't put too much expectation on myself, take little steps, little bits at a time. (Male, restitu- tion, MS)}

Changes in participants' attitudes towards disclosing their memory problems formed a recurrent pattern in the interviews of participants receiving memory rehabilitation. Respondents described becoming more open about their difficulties, as well as the use of memory aids. Acknowledging and discussing their problems within the groups was identified as the main component facilitating this change.

I' m less embarrassed by it. If I forget somebody's name, I just say "I'm sorry, I've got MS, that's how it is". I try not to use MS as an excuse, I try not to apologise for it, but that's how it is. I' $\mathrm{m}$ not going to be mastermind. (Female, compensation, MS)

\section{Benefits of the group setting}

This was a core category across the three programmes. The majority of participants enjoyed working in a group, which they saw as a supportive and non-judgemental context where they could share their difficulties and exchange ideas. To some participants, it was also an opportunity for social interaction and a reminder that, despite their cognitive losses, they could successfully relate to their peers and be a valued group member.

Being with other people who also can't remember you think "Oh, you know, in the grand scheme of thing, I can still function as a person without some of my memories". So it made me less, not so hard on myself, cause it's very hard to let something go. (Female, compensation, TBI)

The group was also viewed as a relaxing environment that afforded its members the opportunity to decide on the level of their involvement. Some respondents reported that they preferred group over individual sessions (participants received an introductory individual session and were also offered one-to-one sessions in case they missed a meeting). The majority, however, agreed that the inclusion of some individual sessions was beneficial, suggesting that they took place further down the line, once some familiarity had developed between the group leader and themselves.

I've actually found that being in the group was more relaxing than being on my own because there was input from everyone...And sometimes in the groups, when you've forgotten something then someone says something that triggers it off! (Female, compensation, TBI)

Participants expressed their opinions on the synthesis of the group they belonged. Being in a mixed aetiology group was seen as an opportunity to look at the same problem from different perspectives.

The group itself was a good mix of people...it was good to have people that didn't have MS but had other problems so that you could see both sides. If people had the same condition, we would all be working along the same path...but different conditions use different solutions. (Male, compensation, MS)

On the contrary, a lack of agreement was observed regarding the inclusion, within the same group, of participants with different levels of memory performance. Opinions appeared to vary based on respondents' perceptions of own abilities in relation to other group members.

I did feel a bit like the old lady of the group...they all seemed much younger than me and did so much better at the memory tests. I was like 20 years older than them! They were in their 30s...but it wasn't a huge thing I' $m$ the kind of person that laughs at these things! (Female, restitution, MS)

Other respondents viewed the presence of people with severe memory problems as a factor potentially inhibiting the flow of the sessions. It seemed important for these respondents to be in a group of people with similar ability levels in order to work at a pace that is suitable for everyone. 
The fact that we all had different problems was really really interesting, it really helped me, but I think if I had been with somebody that was quite severe, I think I would have found that quite irritating after a while. (Female, compensation, TBI)

\section{DISCUSSION}

Participants reported considerable gains, consistent with the aims of holistic memory rehabilitation programmes. In contrast, no statistically significant improvements were found in the outcome measures used in the RCT, apart from an increase in the use of internal memory aids. ${ }^{21}$ Participants' feedback provided a different, more positive picture than was apparent from looking at the quantitative results alone. Respondents agreed that the intervention groups helped them explore the nature and extent of their memory problems, enhanced their sense of confidence and control over the management of memory difficulties, and introduced them to new memory aids and strategies to facilitate their use. These effects were not identified in the interviews of people in the self-help programme.

The benefits of obtaining a better understanding of how memory works were stressed by participants, who called for better information provision by healthcare professionals on the cognitive consequences of BI. Our findings further support earlier recommendations ${ }^{18}$ on the value of measuring improvements in patients' memory knowledge which, to date, is lacking from memory rehabilitation studies. Receiving feedback on their memory performance and interacting with other group members were identified as factors promoting self-awareness. The importance of this finding becomes apparent in the light of studies which identify the management of self-awareness issues as critical to the success of rehabilitation efforts, mediating participants' motivation and engagement with rehabilitation. ${ }^{38} 39$ Together with qualitative evaluations of holistic rehabilitation programmes, our results demonstrate the potential for improvements in this area. ${ }^{40} 41$ It is worth noting, however, that some participants felt unprepared to engage in this process of self-reflection, eventually leading to a participant dropping out of the programme. This finding suggests that clinicians may need to explore patients' coping mechanisms before addressing awareness issues.

Participants learnt to make effective use of the strategies they had in place, or replaced them with new ideas that better fitted their needs and cognitive strengths. These qualitative improvements may not necessarily translate into an increase in the number or frequency of use of memory aids, which might explain why these were not captured by the measures used in the trial. In their study, Evans and Wilson ${ }^{18}$ found that perceived improvements in the use of memory aids were followed by reductions in the actual number of memory aids reported in questionnaires. Taken together, these findings suggest that frequency indices may not be sensitive to changes in the use of memory aids following rehabilitation, offering only a partial picture of issues around their application in day-to-day life.

Respondents acknowledged that rehabilitation did not improve their memory function per se but provided them with the skills to effectively cope with memory problems. Where a reduction of memory failures was reported, it was mainly in relation to prospective memory tasks that allowed the application of memory aids. Despite the encouraging evidence in the field, it has been previously noted that the assessment of memory rehabilitation relies heavily on retrospective memory measures ${ }^{42}$ with only a limited number of studies incorporating ecologically valid prospective tasks. Our analysis indicated that prospective memory is an area which can benefit from memory rehabilitation and, therefore, relevant measures should be included in the evaluation of rehabilitation outcomes.

Although not directly addressed by the programmes, benefits were reported in relation to participants' sense of self-efficacy and control over memory difficulties. Evidence suggests that control beliefs may affect both memory performance and compensatory behaviours. ${ }^{33} 43$ Putting memory aids into practice would appear pointless to users who do not believe in their ability to effectively cope with their memory problems. The value of addressing and evaluating control beliefs as part of cognitive rehabilitation programmes is increasingly acknowledged in the literature. ${ }^{44}$ To date, the few studies that have included control beliefs as a memory training component were conducted on healthy older adults. ${ }^{45}$ There is a need for research which directly addresses control beliefs and evaluates their contribution to the effectiveness of memory rehabilitation interventions for people with neurological disabilities.

Rehabilitation appeared to have contributed to participants' emotional adjustment to the effects of memory impairment. Respondents felt supported in managing memory anxiety and overcoming their embarrassment about using memory aids, a potentially major barrier to their practice. ${ }^{46}$ These changes may not be captured by generic mood questionnaires particularly when they do not provide separate indices for anxiety and depression symptoms. The use of global measures may further hinder detecting these subtle improvements. The benefits reported by participants in this study were very specific and limited to memory-related functions which could explain why they were not identified by the generic outcome measures used in the trial. Indices of activities of daily living, for example, will not necessarily respond to changes in participants' independence at performing memory tasks. Similarly, although a number of global scales of control beliefs are available, it has been recognised that domain-specific measures of perceived control are better predictors of cognitive performance. ${ }^{47}$ The use of measures focusing on the specific cognitive functions targeted by the intervention is therefore recommended to complement those assessing generalised improvements following rehabilitation. 
Our findings highlighted the beneficial effects of a group-based approach to memory rehabilitation. Most participants recognised the combination of a group-based approach, along with one or two individual sessions, as their preferred format. Future research could evaluate this further as, to the best of our knowledge, there is currently a lack of controlled studies examining the effectiveness of group-based over individually administered cognitive rehabilitation. Although participants saw benefits in mixed aetiology groups, differences in cognitive performance were described as a factor potentially undermining the cohesion of the group, diverting the focus from the learning process to individual performance. Being mindful of these issues might be a good practice for clinicians running cognitive rehabilitation groups.

Our results echo those of earlier studies ${ }^{18-20}$ indicating a mismatch between patient-valued outcomes and the information obtained from quantitative outcome measures traditionally used in memory rehabilitation research. This evidence calls for further evaluation of the way we measure outcome in memory rehabilitation to ensure important aspects of patient adaptation process are not missed. The categorical scheme we developed is in agreement with the themes identified in the pilot phase of the trial, ${ }^{20}$ further supporting the validity of the analysis. The use of semistructured interviews enabled richer descriptions and less ambiguous responses, leading to the development of more welldefined categories. The constant comparison analytic process allowed the identification of issues specific to the two intervention programmes. It has to be noted, however, that these qualitative comparisons cannot provide information on the significance of the observed differences. Rather than comparing their effectiveness, we were interested in the patient-perceived effects of all the three programmes as they correspond to integral components of memory rehabilitation commonly delivered in clinical practice. Examining the frequency of occurrence of each category further highlighted the differences between the three programmes. However, the number of responses in each category should not be viewed as a hierarchy but rather as an indication of the prevalence of each category in relation to the rest. Apart from the most frequently cited categories, individual cases contradicting the main patterns were also presented to offer a more rounded picture of the data.

The heterogeneity of our sample reflected researchers' intentions to capture the reality of clinical practice within the context of a pragmatic trial. Although detecting cause and effect relationships between participant characteristics and perceived outcomes was out of the scope of this study, participants' basic demographic and psychometric characteristics were presented to allow readers to evaluate the relevance of this group of patients to their population of interest. The three programmes were comparable in terms of participant age, time since injury/diagnosis, cognitive ability and mood, suggesting that the identified differences between the intervention and the control programmes may be attributable to the specific effects of rehabilitation rather than these characteristics. The analytical approach we followed allowed us to look for patterns across the data set in order to identify intervention-specific benefits; it did not, however, permit an in-depth understanding of possible interconnections between categories within individual interviews. Future studies should focus on investigating and theorising the factors and mechanisms that account for individual differences in perceived outcomes, possibly using bigger samples to facilitate meaningful subgroup analyses.

The findings of this study are based on participants' reports and therefore they are open to biases related to self-evaluation. Respondents' poor self-awareness, as well as their willingness to provide feedback that would satisfy the researchers, may have led to exaggerated and inaccurate descriptions. Although a possible interference of these factors cannot be ruled out, they cannot fully account for the benefits reported in the interviews. Participants provided a balanced account of the programmes identifying both gains, as well as domains that rehabilitation fell short of improving. Participants also reflected on the difficulties associated with appraising the effects of the programmes at this early stage of their recovery. These issues may need to be taken into account by researchers when interpreting rehabilitation outcomes, particularly when self-report measures and interviews are used.

In conclusion, our study suggested that patients with neurological disabilities experienced important benefits following memory rehabilitation which may not be captured by quantitative outcome measures alone. Rehabilitation professionals may want to consider assessing improvements in patients' memory knowledge, selfefficacy, control beliefs and emotional adjustment, using domain-specific measures. Frequency indices may not be sensitive to changes in the use of memory aids, and provide a false picture of patients' progress in effectively managing their memory problems. Patients may benefit from group rehabilitation interventions, but further research is needed before drawing any safe conclusions on their advantage over individual sessions.

Acknowledgements The authors would like to thank all participants for their contribution to the study and Katherine Siu for assisting with the data collection. The research was supported by the NIHR Collaboration for Leadership in Applied Health Research and Care East Midlands (CLAHRC EM). The views expressed are those of the authors and not necessarily those of the NHS, the NIHR or the Department of Health.

Contributors NBC acquired and analysed the data and led the writing of the manuscript. NL contributed to the design of the study and interpretation of the findings, revised and approved the final version before submission.

Funding This research received no specific grant from any funding agency in the public, commercial or not-for-profit sectors.

Competing interests None declared.

Ethics approval The study was approved by the Nottingham Research Ethics Committee 1. 
Provenance and peer review Not commissioned; externally peer reviewed.

Data sharing statement No additional data are available.

Open Access This is an Open Access article distributed in accordance with the Creative Commons Attribution Non Commercial (CC BY-NC 4.0) license, which permits others to distribute, remix, adapt, build upon this work noncommercially, and license their derivative works on different terms, provided the original work is properly cited and the use is non-commercial. See: http:// creativecommons.org/licenses/by-nc/4.0/

\section{REFERENCES}

1. World Health Organisation. International Classification of Functioning, Disability and Health. Genewa, Switzerland: WHO, 2001.

2. Prigatano GP. A history of cognitive rehabilitation. In: Halligan PW, Wade DT, eds. Effectiveness of rehabilitation for cognitive deficits. Oxford: Oxford University Press, 2005:3-11.

3. Wilson BA. Case studies in neuropsychological rehabilitation. Oxford: Oxford University Press, 1999.

4. Lincoln NB, Nair R. Outcome measurements in cognitive neurorehabilitation. In: Stuss DT, Winocur G, Robertson IH. eds. Cognitive neurorehabilitation: evidence and application. 2nd edn. Cambridge: Cambridge University Press, 2008:91-106.

5. Gillespie DC, Bowen A, Chung CS, et al. Rehabilitation for post-stroke cognitive impairment: an overview of recommendations arising from systematic reviews of current evidence. Clin Rehabil 2015;29:120-8.

6. Campbell NC, Murray E, Darbyshire J, et al. Designing and evaluating complex interventions to improve health care. BMJ 2007;334:455-9.

7. Wade DT. Outcome measures for clinical rehabilitation trials: impairment, function, quality of life or value? Am J Phys Med Rehabil 2003;82(Suppl 10):26-31.

8. Craig P, Dieppe P, Macintyre S, et al. Developing and evaluating complex interventions: the new Medical Research Council guidance. BMJ 2008:33:a1655.

9. Streiner DL, Norman GR. Health measurement scales; a practical guide to their development and use. 4th edn. Oxford: Oxford University Press, 2008.

10. Crook THIII, Larrabee GJ. A self-rating scale for evaluating memory in everyday life. Psychol Aging 1990;5:48-57.

11. Sunderland A, Harris JE, Baddeley AD. Do laboratory tests predict everyday memory? A neuropsychological study. J Verbal Learn Verbal Behav 1983;22:341-57.

12. Gilewski MJ, Zelinski EM. Memory functioning questionnaire (MFQ). Psychopharmacol Bull 1988;24:665-70.

13. Chouliara N, Lincoln NB. Developing a questionnaire to assess the outcome of memory rehabilitation for people with neurological disabilities. Int J Ther Rehabil 2015;22:470-8.

14. Popay J, Williams G. Qualitative research and evidence-based healthcare. J R Soc Med 1998;91:32-7.

15. Lewin S, Glenton C, Oxman AD. Use of qualitative methods alongside randomized controlled trials of complex healthcare interventions: methodological study. BMJ 2009;339:3496-503.

16. O' Cathain A, Murphy E, Nicholl J. Why, and how, mixed methods research is undertaken in health services research in England: a mixed methods study. BMC Health Serv Res 2007;7:85-96.

17. Rowle E, Morriss R, Currie G, et al. Research into practice: Collaboration for Leadership in Applied Health and Social Care Research (CLAHRC) for Nottinghamshire, Derbyshire, Lincolnshire (NDL). Implement Sci 2012;3:40.

18. Evans JJ, Wilson BA. A memory group for individuals with brain injury. Clin Rehabil 1992;6:75-81.

19. Quemada JI, Muñoz Céspedes JM, Ezkerra J, et al. Outcome of memory rehabilitation in traumatic brain injury assessed by neuropsychological tests and questionnaires. J Head Trauma Rehabil 2003;18:532-40.

20. das Nair R, Lincoln NB. The effectiveness of memory rehabilitation following neurological disabilities: a qualitative inquiry of patient perspectives. Neuropsychol Rehabil 2013;23:528-45.
21. das Nair R, Lincoln NB. Evaluation of rehabilitation of memory in neurological disabilities: a randomised controlled trial (REMIND). Clin Rehabil 2012;26:894-903.

22. Wilson BA, Clare L, Baddeley AD, et al. The Rivermead Behavioural Memory Test-Extended Version. Bury St. Edmunds, UK: Thames Valley Test Company, 1999.

23. Wilson BA, Moffat N. Clinical management of memory problems. Bury St Edmunds: Chapman \& Hall, 1984.

24. Goldberg RJ, Williams PA. User's guide to the General Health Questionnaire. Windsor: NFER-Nelson, 1988.

25. Coughlan A, Storey P. The Wimbledon Self-Report Scale: emotional and mood appraisal. Clin Rehabil 1988:2:207-13.

26. Nouri F, Lincoln NB. An extended activities of daily living scale for stroke patients. Clin Rehabil 1987;1:233-8.

27. Johnson BR, Onwuegbuzie AJ. Mixed methods research: a research paradigm whose time has come. Educ Res 2004;33: $14-26$.

28. Feilzer MY. Doing mixed methods research pragmatically: implications for the rediscovery of pragmatism as a research paradigm. J Mixed Methods Res 2010;4:6-16.

29. Powell T, Malia K. The brain injury work book, exercises for cognitive rehabilitation. Bicester: Speechmark Publishing, 2003.

30. Waltz CF. Measurement in nursing research. 2nd edn. Philadelphia: Davis, 1991

31. Zhang Y. Wildemuth BM. Qualitative analysis of content. In: Wildemuth BM, ed. Applications of social research methods to questions in information and library science Westport. CT: Libraries Unlimited, 2009:308-19.

32. Krippendorff K. Content analysis: an introduction to its methodology. 2nd edn. London: Sage, 2004.

33. Lachman ME, Bandura M, Weaver SL, et al. Assessing memory control beliefs: the memory controllability inventory. Aging Neuropsychol C 1995;2:67-84.

34. Rourke L, Anderson T. Validity in qualitative content analysis. ETR D 2004;52:5-18. http://dx.doi.org/10.1007/BF02504769

35. Nelson HE, Wilson J. National Adult Reading Test. Chiswick: NFER-Nelson Publishing, 1991.

36. Syder D, Body R, Parker M, et al. Sheffield Screening Test for Acquired Language Disorders. Windsor: NFER-Nelson, 1993.

37. Cavanaugh JC, Feldman JM, Hertzog C. Memory beliefs as social cognition: a reconceptualization of what memory questionnaires assess. Rev Gen Psychol 1998;2:48-65.

38. Prigatano GP, Schacter DL. Awareness of deficit after brain injury. Oxford: Oxford University Press, 1991.

39. Ownsworth T, Clare L. The association between awareness deficits and rehabilitation outcome following acquired brain injury. Clin Psychol Rev 2006;26:783-95.

40. Nilsson C, Bartfai A, Löfgren M. Holistic group rehabilitation-a short cut to adaptation to the new life after mild acquired brain injury Disabil Rehabil 2011;33:969-78.

41. Lexell EM, Alkhed AK, Olsson K. The group rehabilitation helped me readjust to a new life: experiences shared by persons with an acquired brain injury. Brain Inj 2013;27: 529-37.

42. Fleming JM, Shum D, Strong J, et al. Prospective memory rehabilitation for adults with traumatic brain injury: a compensatory training programme. Brain Inj 2005;19:1-10.

43. Berry JM. Memory self-efficacy in its social cognitive context. In: Hess TM, Blanchard-Fields F, eds. Social cognition and aging. San Diego, CA: Academic Press, 1999:70-98.

44. Dawson DR, Winocur G. Psychosocial considerations in cognitive rehabilitation. In: Stuss DT, Winocur G, Robertson IH, eds. Cognitive neurorehabilitation: evidence and application. 2nd edn. Cambridge: Cambridge University Press, 2008:232-50.

45. McDougall GJ, Kang J. Memory self-efficacy and memory performance in older males. Int J Ment Health 2003;2: 131-47.

46. Wilson BA, Watson PC. A practical framework for understanding compensatory behaviour in people with organic memory impairment. Memory 1996;4:465-86.

47. Valentijn SAM, Hill RD, Hooren V, et al. Memory self-efficacy predicts memory performance: results from a 6-year follow-up study. Psychol Aging 2006;21:165-72. 